\title{
Spectral Imaging as a Potential Tool for Optical Sentinel Lymph Node Biopsies
}

\author{
Jack D. O'Sullivan ${ }^{* a}$, Paul R. Hoy ${ }^{\mathrm{b}}$, Harvey N. Rutt ${ }^{\mathrm{a}}$ \\ ${ }^{a}$ Faculty of Physical \& Applied Sciences, University of Southampton, Southampton, SO17 1BJ, UK; \\ ${ }^{\mathrm{b} S}$ School of Medicine, University of Southampton, Southampton, SO17 1BJ, UK
}

\begin{abstract}
Sentinel Lymph Node Biopsy (SLNB) is an increasingly standard procedure to help oncologists accurately stage cancers. It is performed as an alternative to full axillary lymph node dissection in breast cancer patients, reducing the risk of longterm health problems associated with lymph node removal. Intraoperative analysis is currently performed using touchprint cytology, which can introduce significant delay into the procedure. Spectral imaging is forming a multi-plane image where reflected intensities from a number of spectral bands are recorded at each pixel in the spatial plane. We investigate the possibility of using spectral imaging to assess sentinel lymph nodes of breast cancer patients with a view to eventually developing an optical technique that could significantly reduce the time required to perform this procedure. We investigate previously reported spectra of normal and metastatic tissue in the visible and near infrared region, using them as the basis of dummy spectral images. We analyse these images using the spectral angle map (SAM), a tool routinely used in other fields where spectral imaging is prevalent. We simulate random noise in these images in order to determine whether the SAM can discriminate between normal and metastatic pixels as the quality of the images deteriorates. We show that even in cases where noise levels are up to $20 \%$ of the maximum signal, the spectral angle map can distinguish healthy pixels from metastatic. We believe that this makes spectral imaging a good candidate for further study in the development of an optical SLNB.
\end{abstract}

Keywords: Sentinel Lymph Node Biopsy, Multispectral Imaging, Hyperspectral Imaging, Spectral Angle Map, Optical Biopsy

\section{INTRODUCTION}

\subsection{Sentinel lymph node biopsy procedure}

In order to effectively plan treatment and determine prognosis for cancer patients, it is important that the stage of the cancer is correctly assessed. Cancer spreads through the body using the lymphatic system; as such this system plays a crucial role in this staging process. In the case of breast cancer patients, fluids from the tumour region enter the lymphatic system through the axillary lymph nodes. During a standard lumpectomy, it is these nodes which must be assessed in order to determine the level of metastasis.

The Sentinel Lymph Node Biopsy (SLNB) is a standard alternative to the Axillary Lymph Node Dissection (ALND) procedure for determining the extent of this involvement The ALND is a full removal of all lymph nodes in the axillary region, these are then tested for the presence of cancerous cells. The Sentinel Lymph Node (SLN) is the first node to which fluids from the tumour region will drain and as such is the primary gateway for metastases and the purpose of the SLNB is determine whether cancerous cells are present in the SLN.

If it is determined that the SLN contains cancerous cells then a full ALND will be performed to assess the extent of lymphatic involvement. If no cancer is found in the SLN then the remaining axillary lymph nodes can be left intact as there is low probability that metastasis has started to occur ${ }^{1}$. This reduces the risk of long term health problems associated with the removal of lymph nodes, such as lymphedema ${ }^{2}$.

Currently the assessment of the SLN is performed by way of touch print or touch prep (TP) cytology ${ }^{3}$. This cytological test is presently the rate-limiting step in the tumour resection surgery. Where the facilities for performing this test are available onsite, the results can be obtained during the course of the surgery, although a delay of up to an hour is not uncommon and this is time that the patient is anaesthetised and "open" in theatre. If no facilities are available onsite,

*jdo@orc.soton.ac.uk; phone +44 2380593163 
then cases where a full ALND is needed require a follow-up procedure at a delay of possibly weeks or months. The aim of this research is to investigate a possible optical based alternative for the assessment of SLNs in order to reduce the delay currently experienced.

\subsection{Spectral imaging}

Spectral imaging is the process of forming an image by measuring the reflected intensity of light from a number of spectral bands at every pixel in the image plane. Two forms of spectral imaging are commonly referred to, hyperspectral and multispectral. The distinction between hyperspectral and multispectral imaging is not exact; as a guide multispectral imaging typically refers to a situation where a small number ( 2-20) of discrete bands is used whereas hyperspectral imaging uses a large number $(>20)$ of bands, which are typically contiguous. As such each pixel in a hyperspectral image consists of a full spectrum whereas multispectral pixels represent sampling of a spectrum at discrete points (see Figure 1 below).

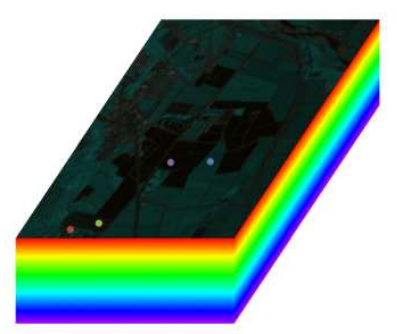

Hyperspectral Image

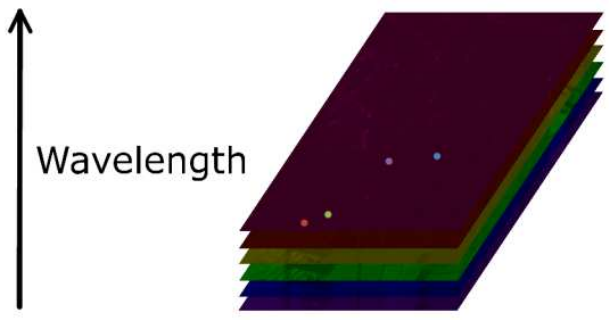

Multispectral Image

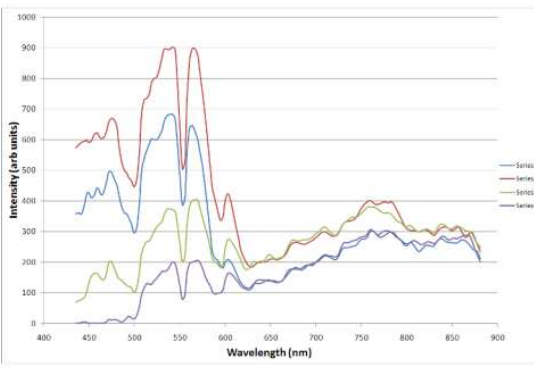

Each pixel has a complete spectrum

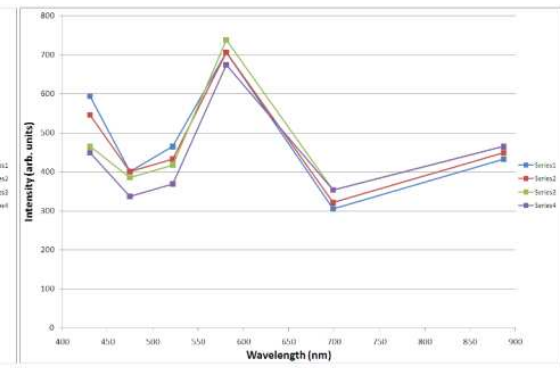

Each pixel has a discretely sampled spectrum

Figure 1 - The same scene in a hyperspectral and multispectral image. The spectral information from four points is shown for each, clearly the hyperspectral image records a complete spectrum whereas the multispectral image records a discretely sampled spectrum

Different materials have different reflection spectra and so by comparing the spectra at each pixel one can assess which image pixels represent similar materials; further by comparing measured spectral data to known spectra of target materials, one can assess the composition of the imaged material. This principle is widely used in Earth remote sensing to assess land cover, for example the Jet Propulsion Laboratory's AVIRIS project (http://aviris.jpl.nasa.gov), it has also been used in a number of other fields such as food contamination detection ${ }^{4}$, solar cell defect detection ${ }^{5}$, illicit substance detection $^{6}$ and disaster management ${ }^{7}$. It can just as easily be applied to biological tissue.

One method of analysing spectral images that is widely used in remote sensing applications, but to our knowledge has not yet been applied to biological studies, is spectral angle mapping ${ }^{8}$. This reduces the data at each pixel to a single value that measures the difference between it and a reference. It is this technique which is the subject of the rest of this paper. 


\section{SPECTRAL ANGLE MAP}

The spectral angle requires a geometric interpretation of a spectrum. Each wavelength or waveband is treated as a coordinate axis in a high-dimensional space; each possible spectrum thus specifies a point in this space and can be thought of as a high-dimensional vector.

As is familiar for three dimensional space, any two vectors, $\mathbf{a}$ and $\mathbf{b}$, in this space will be co-planar, and will diverge from each other at a fixed angle, $\theta$, which can be calculated from the scalar product:

$$
\theta=\arccos \left(\frac{\mathbf{a} \cdot \mathbf{b}}{|\mathbf{a}||\mathbf{b}|}\right) .
$$

This angle, $\theta$, is the spectral angle between two spectra. For exactly coincident spectra, this angle will be 0 and it will increase as the spectra become more dissimilar. Since all values in a spectral image are non-negative, we are only considering arccos in the domain [0,1], this places an upper limit on the spectral angle of $\pi / 2$ radians. It is worth noting that all vectors $k \mathbf{a}$ will subtend the same angle from $\mathbf{b}$. In terms of spectral imaging, this means that the spectral angle is illumination independent, i.e. changes in illumination across the image will not affect the results.

This angle can be calculated for each image pixel (against a standard reference spectrum), thus creating a spectral angle map (SAM).

\section{APPLICATION}

We are working on spectral imaging in the visible and near infrared range (400-1000nm), and so to test the viability of the SAM, we have constructed a dummy image using visible spectra of healthy and metastatic sentinel nodes of breast cancer sufferers reported by Bigio et $\mathrm{al}^{9}$ (see Figure 2).

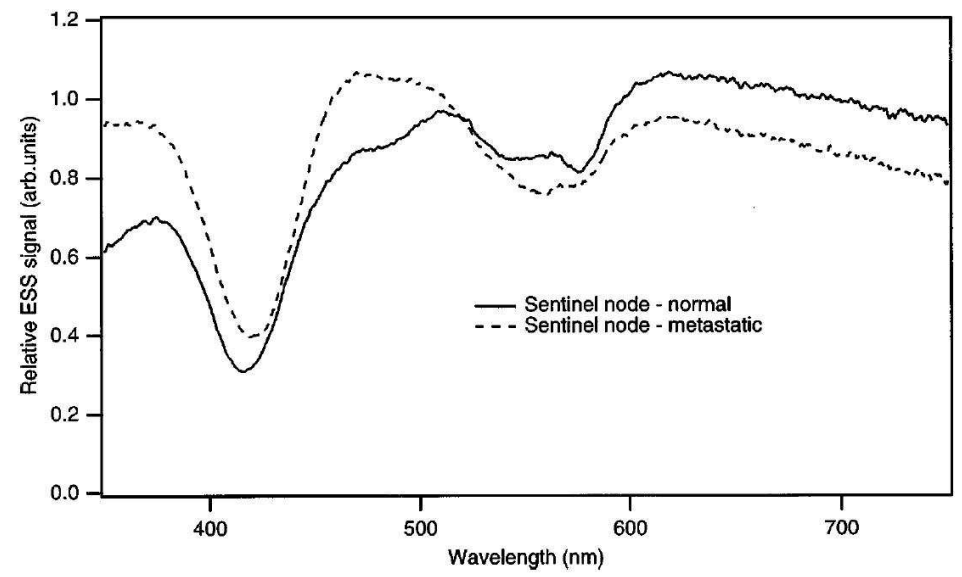

Figure 2 - Diffuse reflectance spectra in the range $\sim 350-750 \mathrm{~nm}$ for normal and metastatic sentinel lymph nodes.

We created a hyperspectral image in which each pixel was either assigned the normal spectrum or the metastatic spectrum according to a binary mask image. Further hyperspectral images were created such that random deviations from the spectrum were added to simulate noise in the measurements. Each measurement of each pixel was randomly adjusted by adding or subtracting a number randomly selected between zero and a given percentage of the maximum signal value. This 'noise level' was increased from $1 \%$ to $20 \%$ in integral increments, each with a new hyperspectral image.

For each image we then created an SAM by calculating the spectral angle of each pixel from a grey reference spectrum, i.e. a spectrum in which every value is the same. Figure 3 below shows the SAMs for two of these hyperspectral images; the image on the left shows the original (all pixels are either filled with the normal, or metastatic spectrum as reported), the image on the right shows the hyperspectral image with the addition of a $20 \%$ noise level. 

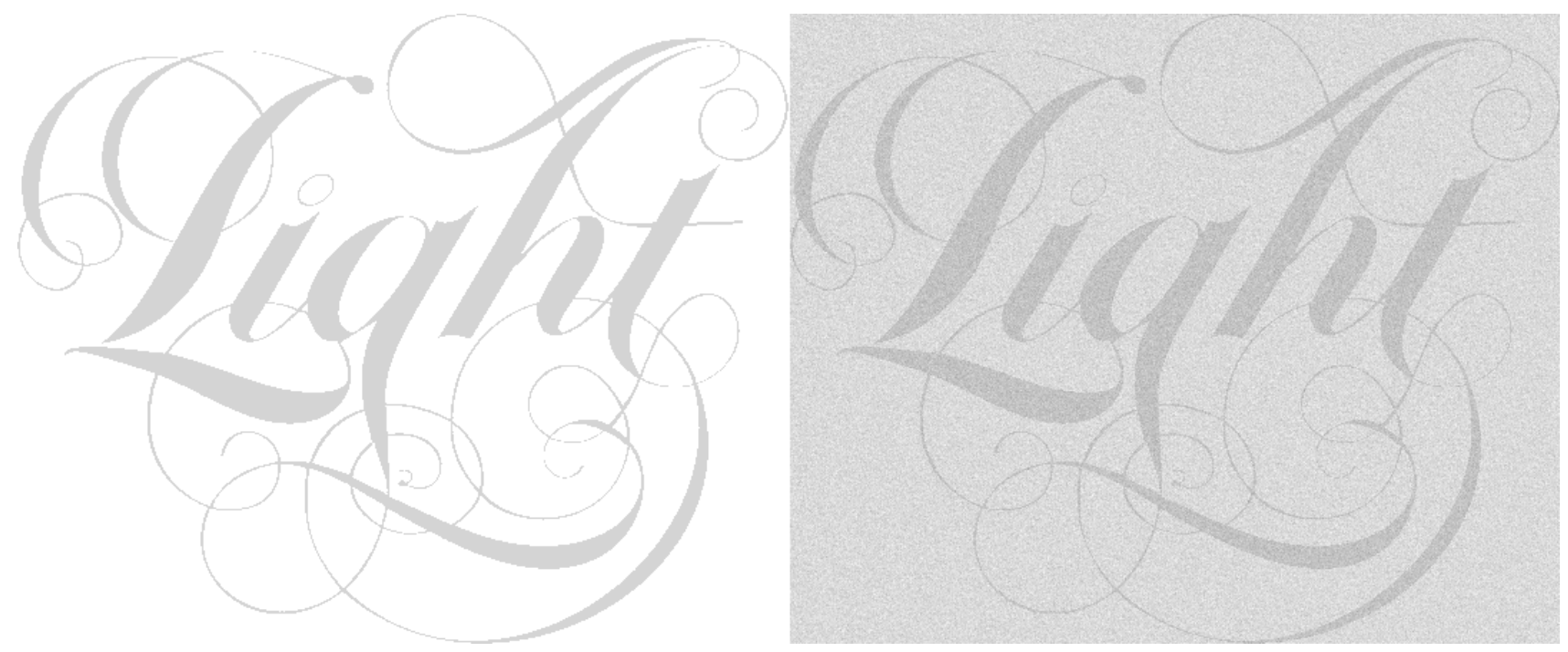

Figure 3 - SAMs of the dummy hyperspectral images, on the left, the original, on the right the image with a $20 \%$ noise level. It is clear that increasing the noise level decreases the separation of the normal and metastatic pixels.

It is evident from Figure 3 that the background (normal) pixels are significantly less well separated from the foreground (metastatic) pixels in the noisy image than they are in the original binary image. In particular the finer spatial details in the foreground are less well defined.

Figures 4-6 show histograms of spectral angle from three of these images, the original image, a $10 \%$ noise level image and a $20 \%$ noise level. On the histogram of the original image we have just two lines at the values of the spectral angles of the normal and metastatic spectra. As we increase the noise, these lines are smeared out into peaks, which gradually merge into each other. This is confirmation of what we would expect to be the case. In the presence noise on the signal, the normal pixels exhibit a range of closely correlated spectra, and the metastatic pixels also exhibit this range. It also confirms what we observed visually from the SAMs, i.e. that the normal and metastatic pixels become less separable in the spectral angle space as the noise was increased.

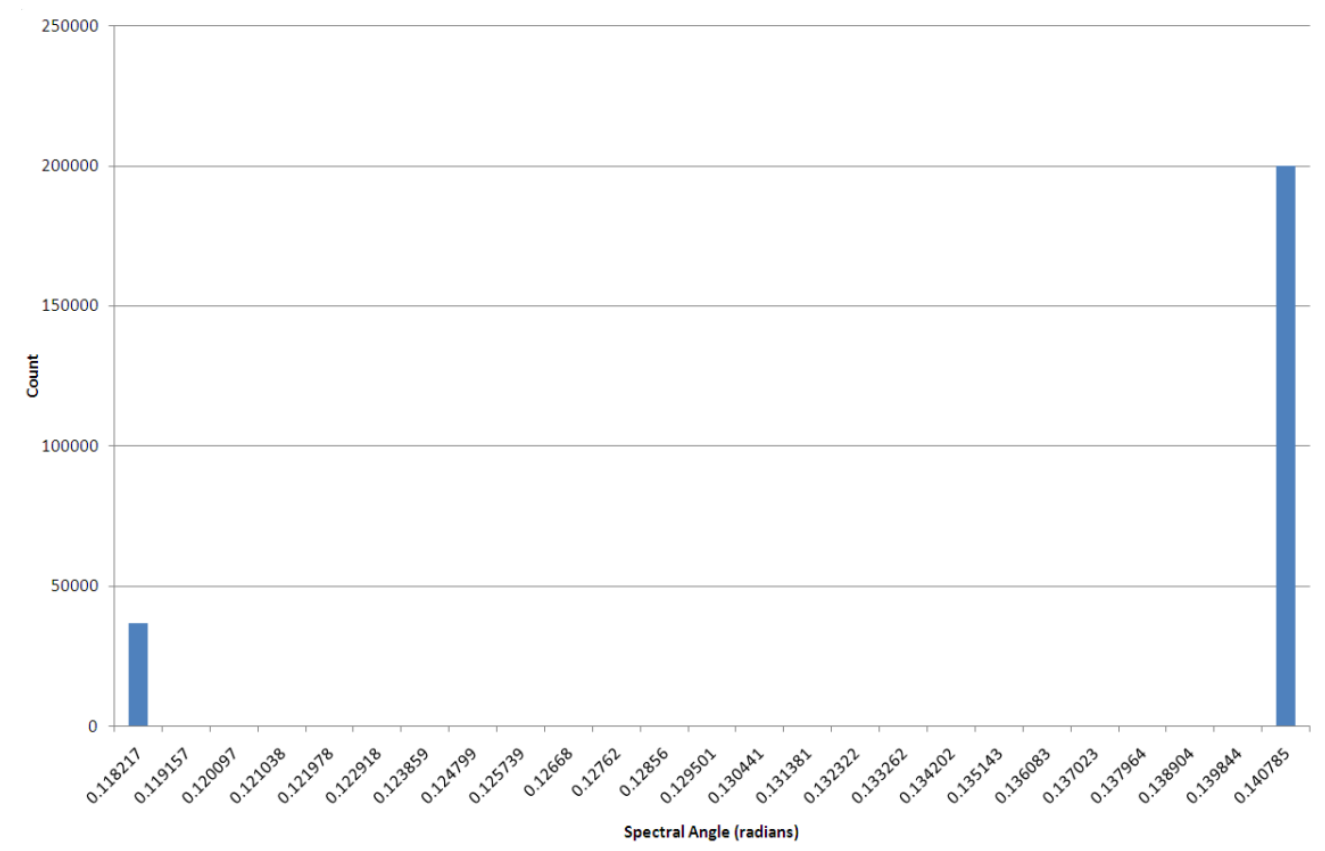

Figure 4 - Histogram of the spectral angles for the original (no noise) dummy hyperspectral image. 


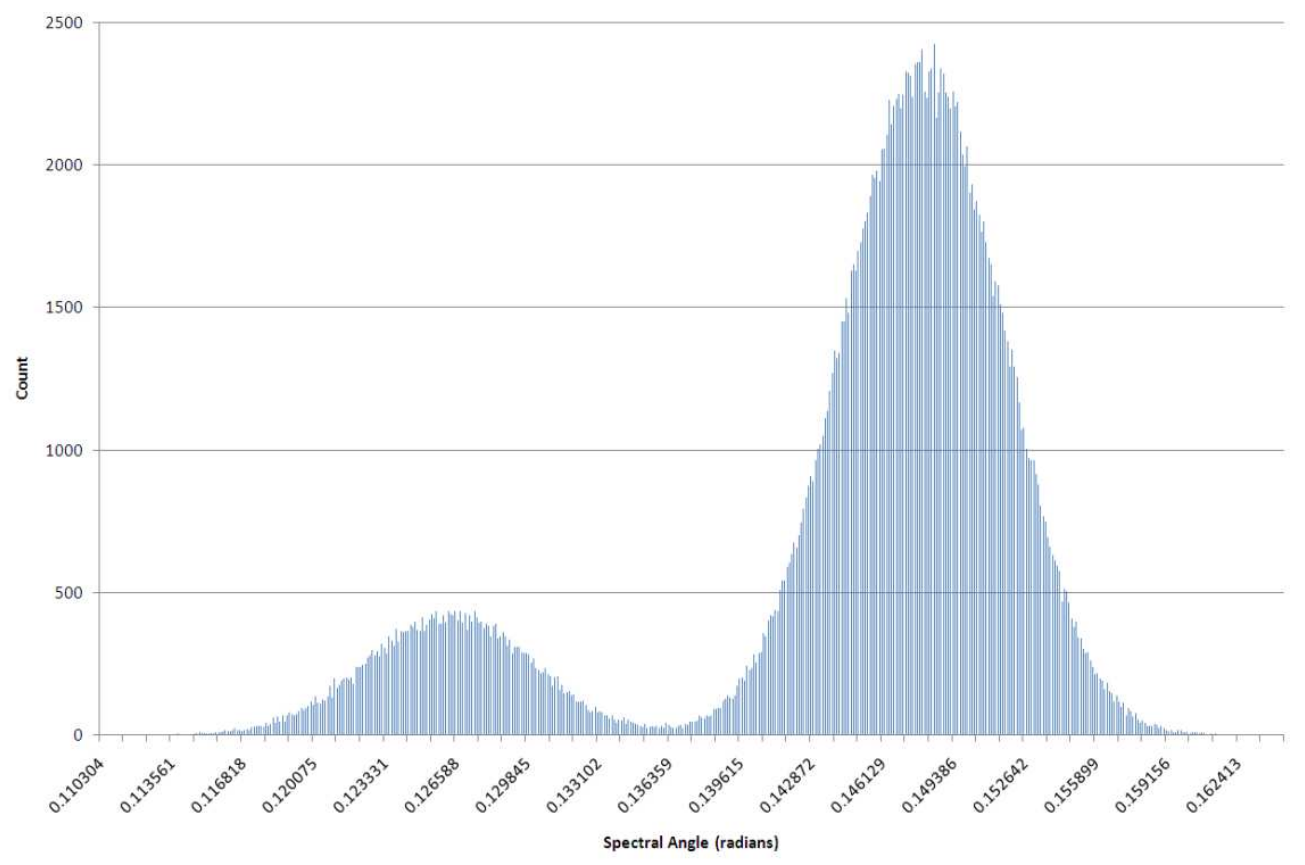

Figure 5 - Histogram of the spectral angles for the $10 \%$ noise level hyperspectral image.

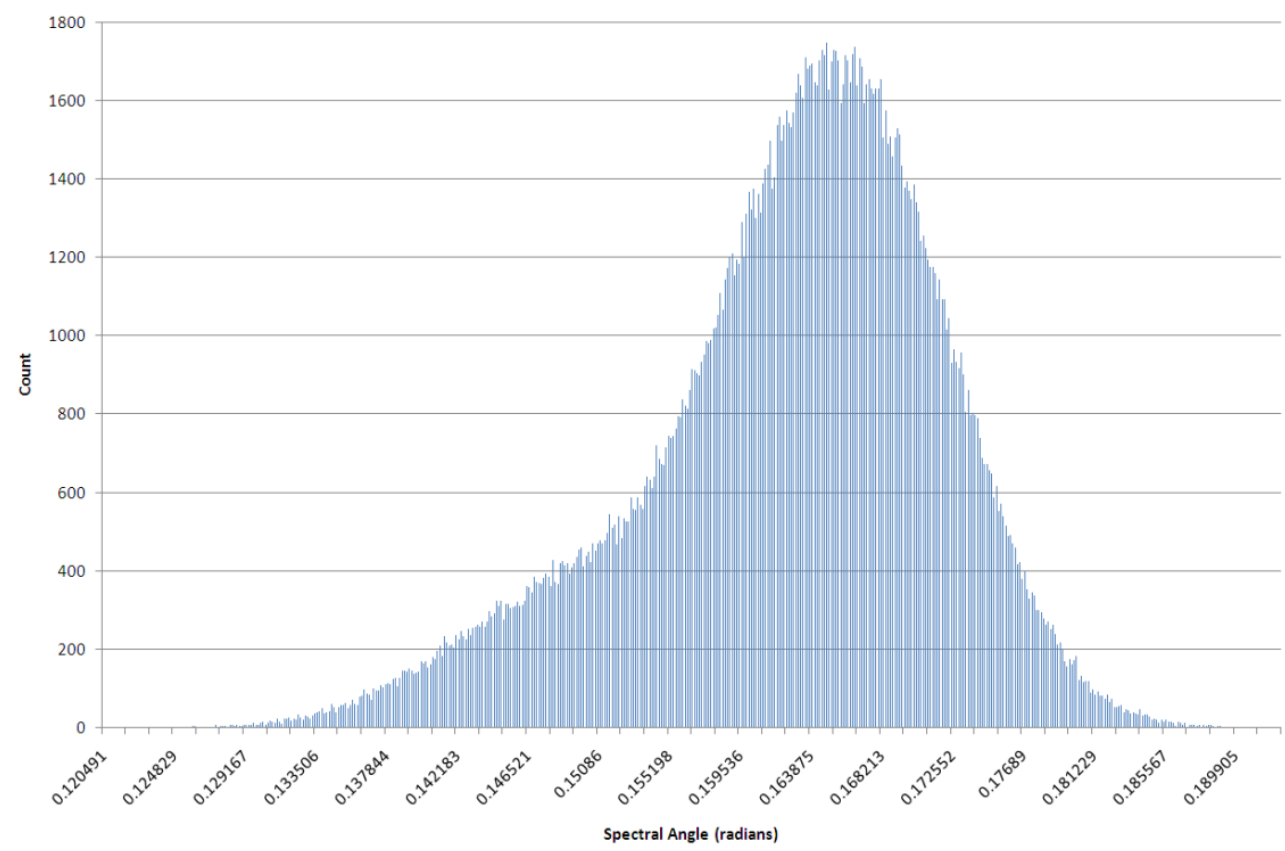

Figure 6 - Histogram of the spectral angles for the $20 \%$ noise level hyperspectral image.

We fitted Gaussian peaks to each of the histograms, the values of the peaks and their widths (shown as error bars) for increasing noise levels, are shown in Figure 7. 


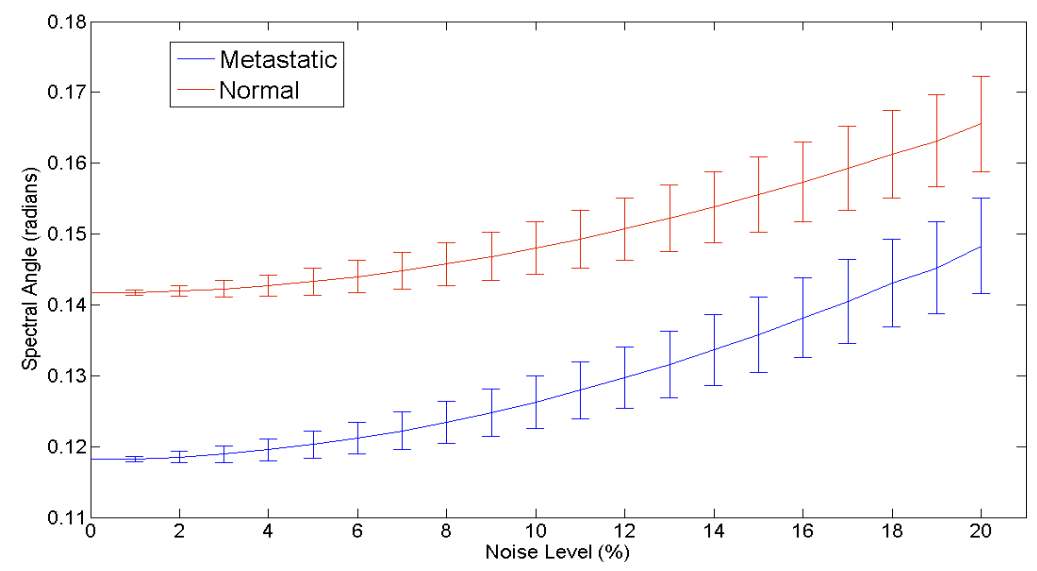

Figure 7 - Graph showing positions and widths of the Gaussian peaks fitted to the histograms of spectral angles for all dummy hyperspectral images. The width of the peak is shown as the error bar at that point.

Using these Gaussian profiles, we classified each pixel as normal or metastatic purely on the basis of its spectral angle. For each pixel, we calculated the probability of it being of part of each peak, it was then randomly assigned to one of the peaks, based on these probabilities. For example, a pixel with a 75\% probability of being a part of the normal peak would have a $75 \%$ chance of being assigned to the normal peak. Figure 8 below shows the results of classification for a low noise level (5\%) and the $20 \%$ noise level image.

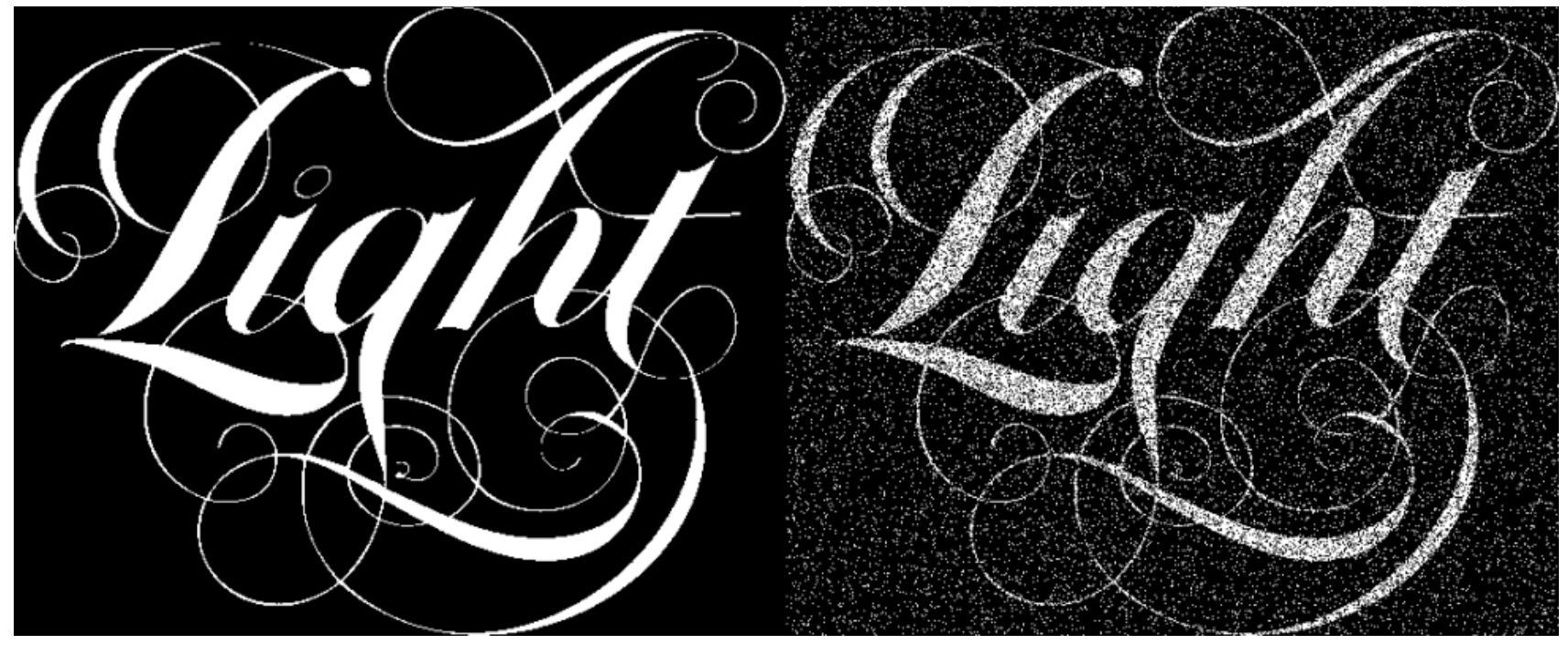

Figure 8 - Classification of pixels as being normal (black) or metastatic (white) based on spectral angle. The left image shows a $5 \%$ noise level spectral angle, the right image shows a $20 \%$ noise level.

The 5\% noise level image shows virtually no misclassification whereas the $20 \%$ noise level image shows a great deal. The foreground is still distinguishable from the background, and the classification seems to be correct for the majority of pixels. We counted the number of misclassified pixels and this is shown below for increasing noise levels in Figure 9. This shows that the number of misclassifications was steady and very low up until noise levels of around 9-10\% before rising steadily. It is around the 9-10\% level at which the peaks on the histograms begin to merge significantly. 


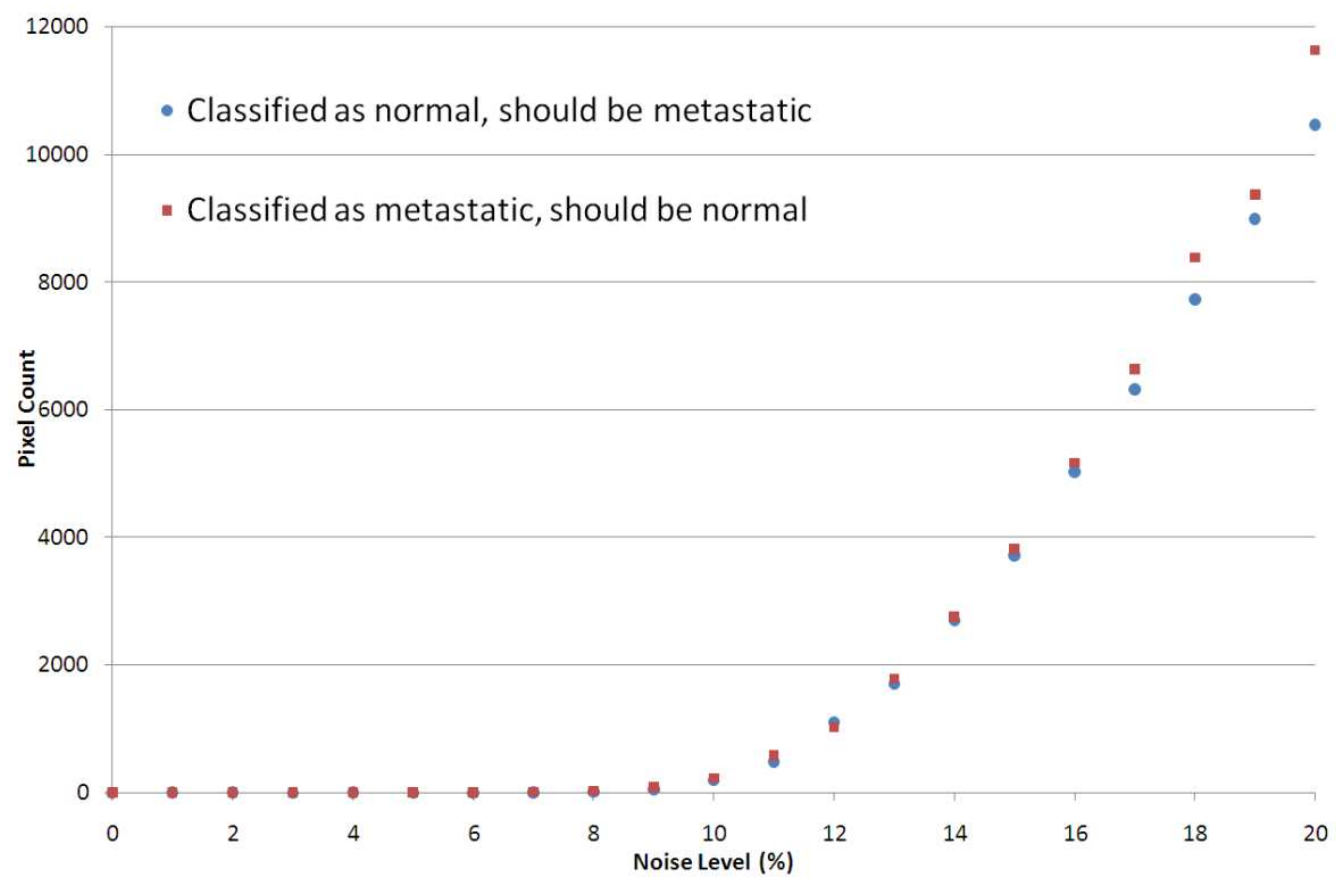

Figure 9 - A graph showing the number of misclassified pixels as a function of noise level, both for pixels that were misclassified as normal and those that were misclassified as metastatic. There are 199853 normal and 36768 metastatic pixels in the original.

Further, we calculated the average spectra for those pixels classified as normal and those classified as metastatic for the $5 \%$ and $20 \%$ noise level image; these are shown together in Figure 10 below.
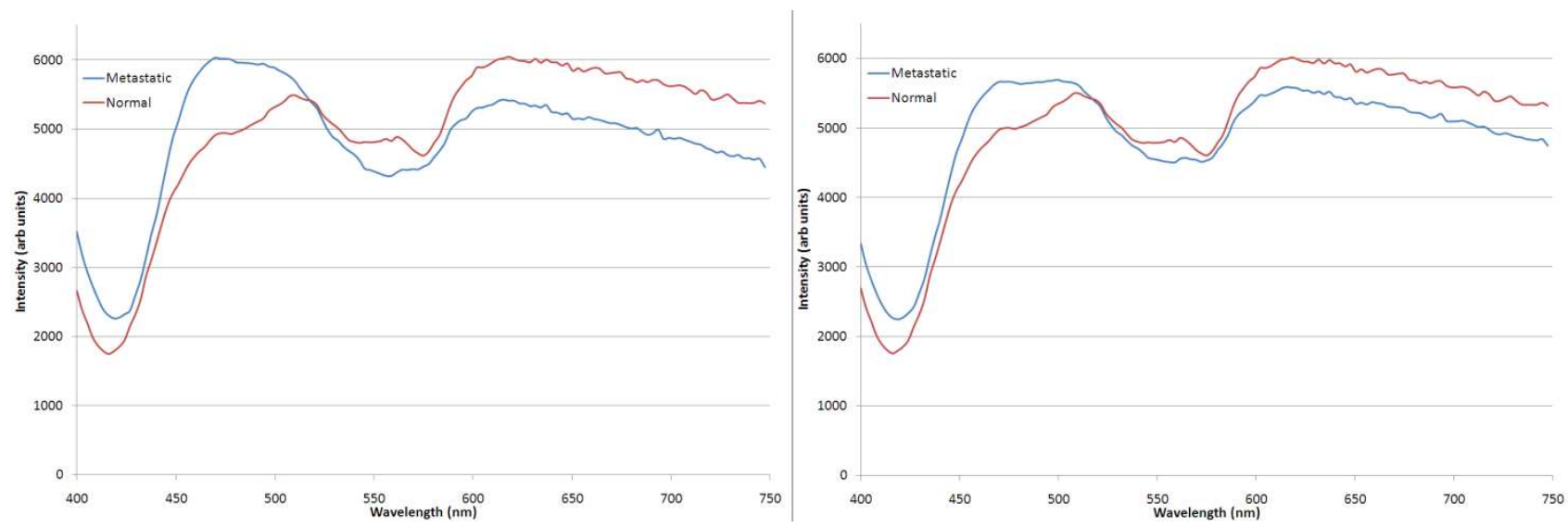

Figure 10 - Average spectra for pixels classified as normal and metastatic. The graph on the left shows the 5\% noise level image, the right hand graph shows those from the $20 \%$ noise level image.

Figure 10 shows that, although still clearly distinguishable, the differences between the spectra are beginning to diminish at high noise levels. In particular, the peak in the metastatic spectrum at around 460nm is significantly reduced, and in the area between that and the start of the decline at around $510 \mathrm{~nm}$ the spectrum becomes much flatter. The separation between the peaks at higher wavelengths is also markedly reduced. It is noted that the normal spectrum is much less changed than the metastatic. 


\section{CONCLUSION}

The spectral angle map clearly allows us to distinguish between the normal and metastatic spectra. Increasing the noise level on the image makes the distinction between normal and metastatic pixels less clear, as the histograms in figures 4-6 demonstrate. However, Figure 7 clearly shows that the spectral angles remain quantifiably separable, even at high noise levels.

The limits on this separability have been explored somewhat further by assessing how well the pixels are classified as being normal or metastatic purely on the basis of their distance in metric space from the peaks of the Gaussian curves present on the histogram. We find that up to around $10 \%$, the misclassification rate is very low but that it climbs steadily as the image gets noisier thereafter. The number of misclassifications is broadly similar for both normal and metastatic (the difference can be accounted for by pixels no longer being classified as either as they have spectral angles far removed from either peak), however this has more impact on the average calculated metastatic spectrum.

There are approximately 5 normal pixels for every metastatic pixel in the original image; we would anticipate that in a real world situation there would be less cancerous tissue in a node than normal tissue. This means however, that every misclassified pixel affects the metastatic spectrum a lot more than the normal spectrum, so whilst the differences between them are changing, as demonstrated in Figure 10, most of this can be attributed to the metastatic spectrum becoming more like the normal.

These results, based as they are on "dummy" data, are very preliminary, and much work remains to be done; however, we believe that they demonstrate that the SAM shows much potential for being the basis of a rigorous and automated classification system, which itself could lead to the development of an optical system for performing SLNBs.

\section{ACKNOWLEDGEMENT}

The authors would like to thank Mr Richard Sainsbury at the School of Medicine, University of Southampton, for sharing his expertise on the subject of breast cancer treatment and specifically the sentinel lymph node biopsy.

\section{REFERENCES}

[1] Howard, S., et al., "Sentinel node biopsy in the staging of breast cancer". American journal of surgery. 176(4): p. 305-310.(1998)

[2] McMasters, K.M., et al., "Sentinel Lymph Node Biopsy for Breast Cancer: A Suitable Alternative to Routine Axillary Dissection in Multi-Institutional Practice When Optimal Technique Is Used". J Clin Oncol. 18(13): p. 2560-2566.(2000)

[3] Rubio, I., et al., "Use of touch preps for intraoperative diagnosis of sentinel lymph node metastases in breast cancer". Annals of Surgical Oncology. 5(8): p. 689-694.(1998)

[4] Park, B., et al., "Contaminant Classification of Poultry Hyperspectral Imagery using a Spectral Angle Mapper Algorithm". Biosystems Engineering. 96(3): p. 323-333.(2007)

[5] Li, Q.L., et al., "Detection of physical defects in solar cells by hyperspectral imaging technology". Optics and Laser Technology. 42(6): p. 1010-1013.(2010)

[6] Ng, P.H.R., et al., "Detection of illicit substances in fingerprints by infrared spectral imaging". Analytical and Bioanalytical Chemistry. 394(8): p. 2039-2048.(2009)

[7] Jusoff, H.K., "Pixel-based airborne hyperspectral sensing technique for search-and-rescue of the missing RMAF NURI helicopter in Genting-Sempah, Malaysia". Disaster Prevention and Management. 19(1): p. 88-102.(2010)

[8] Kruse, F.A., et al., "The Spectral Image-Processing System (SIPS) - Interactive Visualization and Analysis of Imaging Spectrometer Data". Remote Sensing of Environment. 44(2-3): p. 145-163.(1993)

[9] Bigio, I.J., et al., "Diagnosis of breast cancer using elastic-scattering spectroscopy: preliminary clinical results". Journal of Biomedical Optics. 5(2): p. 221-228.(2000) 